$12-1-2020$

\title{
Investir dans le changement social et comportemental est rentable pour augmenter l'utilisation de la contraception moderne au Togo
}

Breakthrough RESEARCH

Follow this and additional works at: https://knowledgecommons.popcouncil.org/departments_sbsr-rh How does access to this work benefit you? Let us know!

\section{Recommended Citation}

Breakthrough RESEARCH. 2020. "Investir dans le changement social et comportemental est rentable pour accroître l'utilisation de la contraception moderne au Togo," Breakthrough RESEARCH Sommaire de Recherche. Washington, DC: Population Council. 


\section{Investir dans le changement social et comportemental est rentable pour accroître l'utilisation de la contraception moderne au Togo}

Les interventions de changement social et comportemental (CSC) sont généralement considérées comme un élément essentiel des services de santé de haute qualité, mais les lacunes dans les informations sur le coût et l'impact du CSC signifient que les décideurs ont sous-estimé la valeur de la CSC pour contribuer à l'amélioration des résultats de la santé. Pour résoudre ce problème, Breakthrough RESEARCH a tiré parti des évidences de 153 études sur l'impact de la planification familiale (PF) CSC et de 70 études sur les coûts du CSC pour évaluer le rapport coût-efficacité de la programmation du CSC pour la PF dans le cadre de son analyse de rentabilisation pour investir dans le changement social et comportemental pour la planification familiale.

\section{Conclusions générales}

Une revue de la littérature a révélé que le CSC augmente I'utilisation de la contraception moderne. Des études montrent que le CSC influence positivement les déterminants intermédiaires qui ont un impact sur l'utilisation de la contraception moderne, tels que les attitudes, la communication avec les partenaires et autres. La force de l'impact varie selon le type d'intervention CSC et le résultat intermédiaire visé (Figure 1).

Les résultats indiquent que les interventions FP CSC sont très rentables. Le coût par année de vie corrigée de l'incapacité $(A V C I)^{1}$ évitée est une mesure courante pour évaluer la rentabilité. Des scénarios d'investissement CSC ont été modélisés en Guinée, au Niger, au Togo et en Zambie. Dans chaque pays, le coût par $\mathrm{AVCl}$ évitée qui en résulte indique que les investissements CSC pour la PF sont très rentables sur la base des références de l'Organisation Mondiale de la Santé (OMS).

Le doublement des investissements FP CSC permet aux programmes de lutter contre l'équité et de s'attaquer aux obstacles plus complexes à l'utilisation de la contraception moderne. Les investissements CSC supplémentaires axés sur la communication interpersonnelle (CIP), les activités de sensibilisation communautaire et les ensembles multi-composants d'interventions CSC au Togo et au Niger ont augmenté I'utilisation attendue de la contraception moderne et étaient toujours rentables. .

\section{FIGURE 1 : VOIES D'IMPACT DU CSC VERS UNE UTILISATION ACCRUE DES CONTRACEPTIFS MODERNES (MISE À JOUR)}

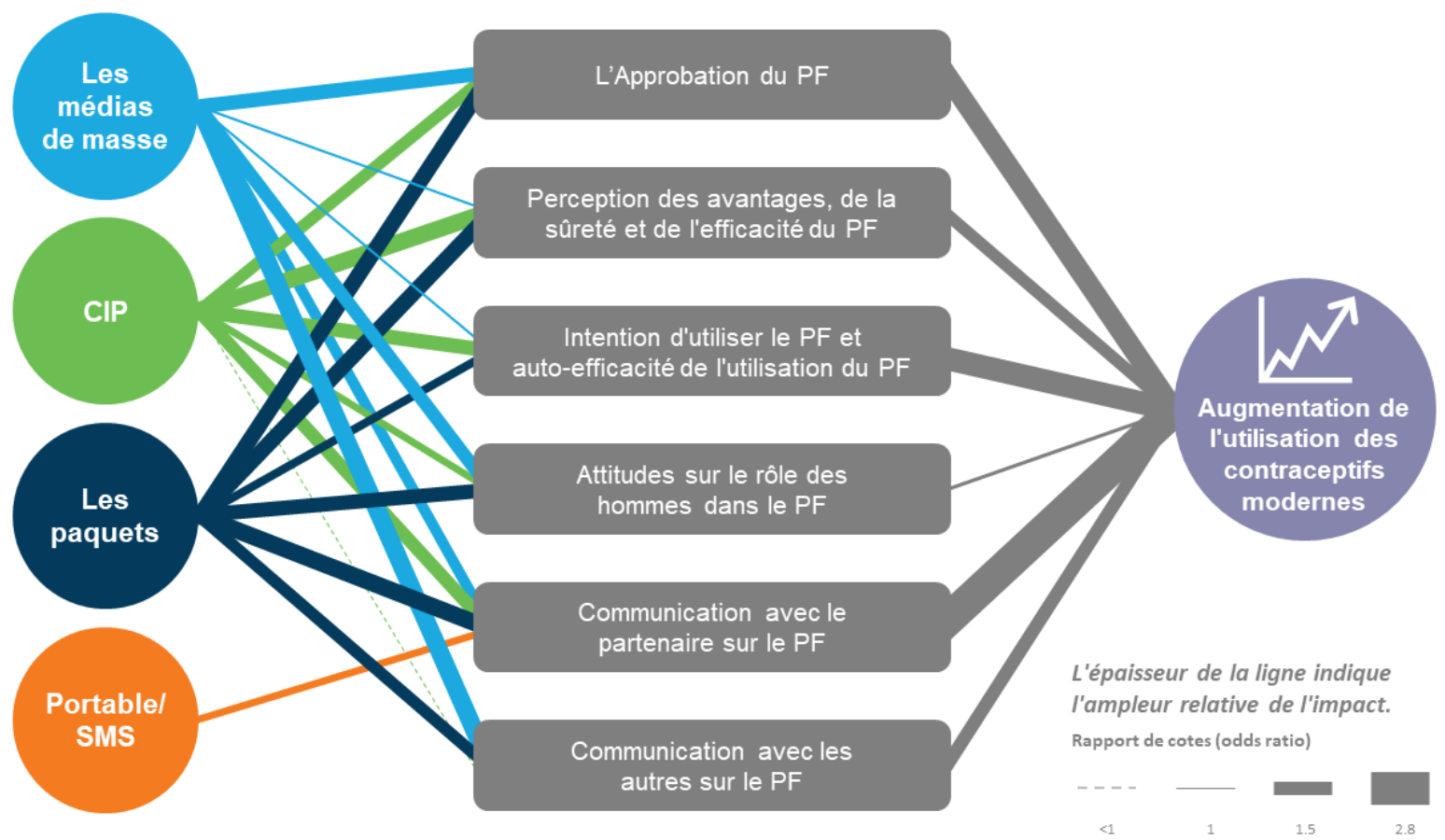

Ce graphique incorpore les données de 153 études, dont 23 études supplémentaires depuis la publication de l'analyse de rentabilisation initiale de la PF pour le CSC en Octobre 2019. 


\section{Csc pour la PF: Un investissement rentable au Togo}

L'analyse de rentabilisation de Breakthrough RESEARCH a modélisé la rentabilité du CSC au Togo en utilisant deux scénarios d'investissement pour évaluer l'impact de la programmation du CSC sur l'utilisation de la contraception moderne.

1. Le scénario du plan national était basé sur les investissements prévus documentés dans le Plan d'Action National Budgetisé de Plantification Familiale 2017-2022 du Togo. Le plan national indiquait un investissement cumulatif sur cinq ans d'environ 3,6 millions de dollars en CSC pour la PF.

2. Un scénario de double investissement a examiné les effets du doublement du total du plan national du Togo à 7,4 millions de dollars avec l'augmentation des investissements ciblant les activités de sensibilisation communautaire et les paquets CSC pour les jeunes qui comprenaient la CIP.

\section{Impact}

Au Togo, le taux de prévalence contraceptive moderne (TPCM) 2020 était de 20,9\%. Sur cinq ans (2021-2025), les investissements CSC basés sur le plan national devraient entraîner une augmentation de 4,8 points de pourcentage du TPCM, environ 124000 grossesses non désirées évitées et $16000 \mathrm{AVCl}$ maternelles sauvées. ${ }^{2}$ Le scénario de double investissement a généré un pourcentage de 7,3 gain de points, évitant environ 187000 grossesses et économisant près de $25000 \mathrm{AVCl}$ maternelles.

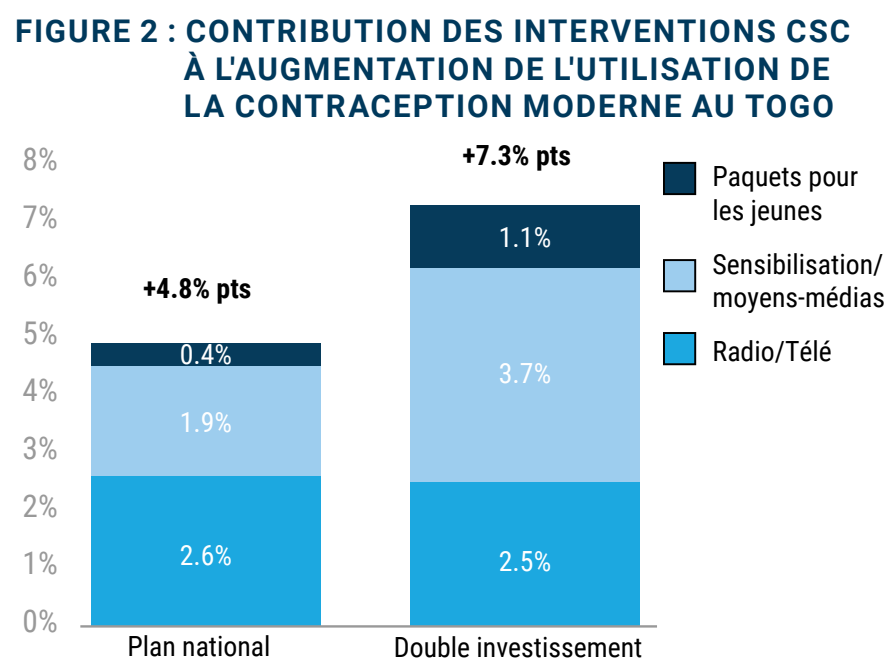

\section{Coût}

Outre les coûts d'investissement du CSC, les coûts directs de prestation de services pour les utilisateurs supplémentaires de contraception moderne se traduisent par un coût total estimé de 6,0 millions de dollars pour le scénario du plan national et de 11,8 millions de dollars pour le scénario de double investissement. ${ }^{3}$

\section{Cost-effectiveness}

Les résultats pour les deux scénarios de mise à l'échelle de I'intervention CSC sont considérés comme très rentables sur la base de la norme de l'OMS de moins d'une fois le produit intérieur brut (PIB) par habitant au Togo de 679,4 USD. Par rapport au PIB de référence moyen pour les pays à revenu intermédiaire d'Afrique Subsaharienne (ASS) à 1612 dollars, le rapport coût-efficacité de l'investissement est encore plus frappant.

Le scénario de double investissement a un coût plus élevé par $\mathrm{AVCl}$ évité car les investissements supplémentaires ont été dirigés vers des interventions plus coûteuses visant à atteindre ceux qui n'ont pas accès aux médias de masse et à surmonter les obstacles plus difficiles à l'utilisation de la PF en s'engageant dans la CIP.

\section{FIGURE 3 : RÉSULTATS RAPPORT COÛT-EFFICACITÉ AU TOGO}

PIB moyen par habitant en Afrique subsaharienne-

seuil de "très efficace par rapport au coût"

$\$ 1,600$

$\$ 1,400$

$\$ 1,200$

$\$ 1,000$

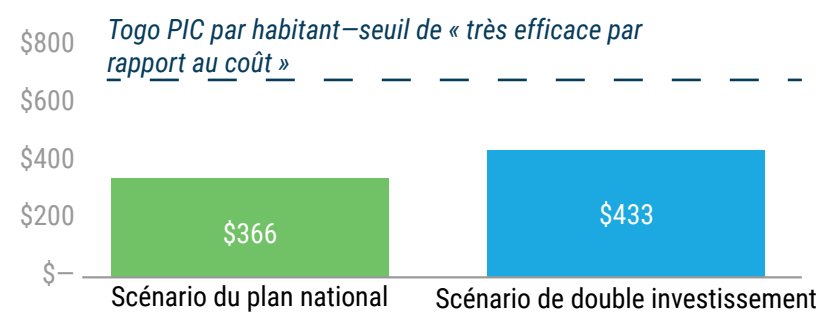

${ }^{1}$ « Une AVCI peut être considérée comme une année perdue de vie« saine ». La somme des AVCI dans la population, ou le fardeau de la maladie, peut être considérée comme une mesure de l'écart entre l'état de santé actuel et une situation sanitaire idéale où toute la population vit jusqu'à un âge avancé, sans maladie ni incapacité (OMS, (WHO, https://www.who.int/healthinfo/global_burden_disease/metrics_ daly/en/, date consultée le 29 octobre 2019).

'L'augmentation estimée de la prévalence contraceptive moderne s'ajoute aux augmentations annuelles dues aux interventions non-CSC.

${ }^{3}$ Sur la base des estimations des coûts médians à partir des coûts unitaires mondiaux et des scénarios de mise à l'échelle supposés; les coûts réels au Togo peuvent varier.

${ }^{4}$ Le seuil de I'OMS pour une intervention hautement rentable est un coût par $\mathrm{AVCl}$ évitée inférieur à une fois le produit intérieur brut par habitant. (Commission de I'OMS sur la macroéconomie et la santé 2001). Comparés à d'autres interventions de santé, ces résultats se situent dans la fourchette moyenne du coût par AVClévitée (Horton et al.2017).

\section{Breakthrough RESEARCH}

Cette activité est mise en œuvre dans le cadre de Breakthrough RESEARCH. Breakthrough RESEARCH suscite un changement social et comportemental (CSC) en menant une recherche et une évaluation de pointe et en promouvant des solutions basées sur des données probantes en vue d'améliorer les programmes de santé et de développement à travers le monde.
Population Council

4301 Connecticut Avenue, NW | Suite 280 | Washington, DC 20008

Tel: +1 2022379400 | breakthroughresearch@popcouncil.org

breakthroughactionandresearch.org

Suggested citation: Breakthrough RESEARCH. 2020. "Investir dans le changement socia

et comportemental est rentable pour accroitre l'utilisation de la contraception moderne

au Togo," Breakthrough RESEARCH Sommaire de Recherche. Washington DC: Population Council. 